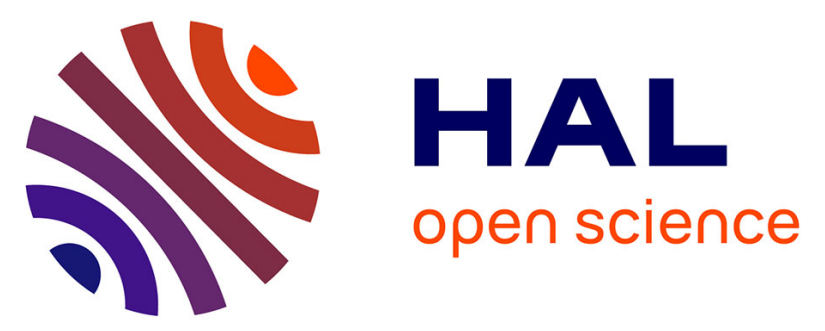

\title{
Sur la présence d'ursus arctos au début de l'Holocène dans le Moyen Atlas (Maroc) - datations et implications paléoenvironnementales
}

Michel Fontugne, Aïcha Oujaa, Brahim Ouchaou, Lahcen Gourari, Samir

Zouhri, Christophe Moreau, Evelyne Kaltnecker, Jean pascal Dumoulin, Michael Werner, Mohammed Benabdelhadi

\section{To cite this version:}

Michel Fontugne, Aïcha Oujaa, Brahim Ouchaou, Lahcen Gourari, Samir Zouhri, et al.. Sur la présence d'ursus arctos au début de l'Holocène dans le Moyen Atlas (Maroc) - datations et implications paléoenvironnementales. Quaternaire, 2012, RQM5. Fès 2009, 23 (1), pp.157-161. 10.4000/quaternaire.6209 . hal-02468900

\author{
HAL Id: hal-02468900 \\ https://hal.science/hal-02468900
}

Submitted on 19 Oct 2020

HAL is a multi-disciplinary open access archive for the deposit and dissemination of scientific research documents, whether they are published or not. The documents may come from teaching and research institutions in France or abroad, or from public or private research centers.
L'archive ouverte pluridisciplinaire HAL, est destinée au dépôt et à la diffusion de documents scientifiques de niveau recherche, publiés ou non, émanant des établissements d'enseignement et de recherche français ou étrangers, des laboratoires publics ou privés. 


\title{
SUR LA PRÉSENCE D'URSUS ARCTOS AU DÉBUT DE L'HOLOCÈNE DANS LE MOYEN ATLAS (MAROC). DATATIONS ET IMPLICATIONS PALÉOENVIRONNEMENTALES
}

\author{
Michel FONTUGNE ${ }^{1}$, Aïcha OUJAA ${ }^{2}$, Brahim OUCHAOU ${ }^{3}$, Lahcen GOURARI ${ }^{4}$, \\ Samir ZOUHRI ${ }^{5}$, Christophe MOREAU ${ }^{6}$, Evelyne KALTNECKER ${ }^{1}$, \\ Jean Pascal DUMOULIN ${ }^{6}$, Michael WERNER ${ }^{7} \&$ Mohammed BENABDELHADI $^{8}$
}

\begin{abstract}
RÉSUMÉ
Dans le Moyen Atlas, près de Boulmane les formations calcaires d'El Minchare abritent une grotte profonde (Ifri Oussaïd) s'ouvrant au-dessus du plateau à $2075 \mathrm{~m}$ d'altitude. Les fouilles de ce site ont livré, de 2005 à 2007, des charbons associés à plusieurs ossements dont au moins 156 restes d'ours brun (Ursus arctos) appartenant à cinq individus. L'étude paléontologique atteste d'un ours de petite taille (Ouchaou, 2008). Les niveaux à charbons de bois scellés dans de petits planchers stalagmitiques situés au-dessus des restes ont donné des dates allant de $5220 \pm 30$ à $5865 \pm 30$ ans ${ }^{14} \mathrm{C}$ BP. La datation obtenue directement sur un radius d'ours est de $7300 \pm 40$ ans ${ }^{14} \mathrm{C} \mathrm{BP}$. Cette date, replacée dans le contexte climatique local, confirme la présence d'Ursus arctos au début de l'Holocène moyen en Afrique du Nord, mais souligne aussi l'ampleur des changements environnementaux dans le Moyen Atlas depuis cette époque et leur influence potentielle sur la disparition d'Ursus arctos de cette région.
\end{abstract}

Mots-clés: Ursus arctos, Moyen Atlas, datations radiocarbone

\section{ABSTRACT}

ON THE OCCURRENCE OF URSUS ARCTOS DURING THE EARLY HOLOCENE IN THE MIDDLE ATLAS (MOROCCO). DATING AND PALAEOENVIRONMENTAL IMPLICATIONS

Near Boulmane city, in the limestone formation of El Minchare, the Ifri Oussaïd cave open over the Middle Atlas plateau at $2075 \mathrm{~m}$ high. From 2005 to 2007 excavations provided charcoal associated to bone remains mainly belonging to Ursus arctos, at last 156 identified bones from five bears. Paleontological studies describe bear of small size (Ouchaou, 2008). Datations of charcoal from stalagmitic floors above bear bone layer give ages between $5220 \pm 30$ and $5865 \pm 30 \mathrm{yrs}{ }^{14} \mathrm{C} \mathrm{BP}$, while the collagen of a radius bone was dated at $7300 \pm 40 \mathrm{yrs}{ }^{14} \mathrm{C} \mathrm{BP}$. This date, taking in account the local climatic pattern, confirms the presence of Ursus arctos at the beginning of the Holocene period in North Africa but also point out to the importance of environmental changes in the Middle Atlas and their potential importance for the extinction of Ursus arctos.

Keywords: Ursus arctos, Middle Atlas, radiocarbon datings

\section{1 - INTRODUCTION}

La présence de l'ours en Afrique du Nord a, depuis longtemps, suscité de nombreuses discussions (Arambourg, 1933; Ennouchi, 1957; Michaux \& Thevenot, 2007). Si, maintenant, sa présence au Maghreb depuis le Pliocène (Geraads, 1997) est bien établie, la date de son extinction reste imprécise et les influences respectives des causes anthropiques ou climatiques restent entière- ment à établir (Hamdine et al., 1998; Ouchaou \& Amani, 2002). Une compilation de Michaux et Thevenot (2007) retrace l'histoire récente de l'ours au Maghreb. Une cinquantaine de sites, depuis la Tunisie jusqu'au Maroc central, la plupart sur la façade maritime de l'Atlas, sont énumérés avec une référence à l'espèce et une attribution à une période géologique allant du Pléistocène inférieur à l'Holocène voire la période historique. Cette synthèse rassemble également les onze datations radiocarbone

\footnotetext{
${ }^{1}$ Laboratoire des Sciences du Climat et de l'Environnement - UMR CEA/CNRS/UVSQ, F-91198 GIF-SUR-YVETTE cedex. Courriel: Michel.Fontugne@lsce.ipsl.fr

${ }^{2}$ Institut National des Sciences de l'Archéologie et du Patrimoine, Rabat, MAROC.

${ }^{3}$ Université Moulay Ismaïl, Faculté des Sciences, Département de Géologie, B.P. 11201, Zitoune, Meknès, MAROC.

${ }^{4}$ Faculté des Sciences Dhar El Mahraz, Fès, MAROC.

${ }^{5}$ Faculté des Sciences Aïn Chok, Casablanca, MAROC.

${ }^{6}$ LMC14, UMS ARTEMIS, F-91190 SACLAY.

${ }^{7}$ Université Paris XII, F-94010 CRÉTEIL

${ }^{8}$ LGRE, Faculté des Sciences et Techniques, Fès, MAROC.
} 
(de 37 à 1,2 ka ${ }^{14} \mathrm{C} \mathrm{BP}$ ) obtenues sur des restes osseux d'ursidés conservés aux musées d'Alger, Rabat, Lyon, Aix-en-Provence et Marseille. Michaux et Thevenot (2007) sur la base des datations radiocarbone font remonter sa disparition au milieu du $\mathrm{I}^{\mathrm{er}}$ millénaire après J.-C. Cependant l'absence de fossiles entre 7,2 ka BP et $1,5 \mathrm{ka}$ BP pose la question de l'origine locale de ces ours récents, ou bien les derniers ours provenaient-ils d'autres régions plus lointaines pour alimenter des spectacles de cirque? L'analyse des reconstitutions paléo-environnementales, dont notamment celles de la végétation, peut fournir un élément de réponse à l'absence de fossile entre $7,2 \mathrm{ka} \mathrm{BP}$ et le premier millénaire de notre ère.

Une autre question posée par la compilation effectuée par ces auteurs est le problème de la co-existence, au début de l'Holocène, d'individus de grande taille et d'individus de petite taille seuls présents à l'Holocène supérieur. Au Maroc, les comparaisons ostéométriques montrent que les ours des niveaux néolithiques et/ou protohistoriques étaient de stature réduite par rapport à ceux du Paléolithique et de l'Epipaléolithique (Ouchaou, 2008), relativement plus imposants que les ours décrits par Hamdine et al. (1998) dans un site d'âge Historique. Ces comparaisons semblent indiquer une diminution progressive de la taille des ours depuis le Pléistocène, phénomène biologique observé dans plusieurs lignées avant leurs extinctions (Michaux \& Thevenot, 2007).

La découverte de nouveaux ossements d'Ursus arctos (Benabdelhadi et al., 2005) permet de prolonger l'étude et la discussion engagée par Michaux et Thevenot (2007) et Ouchaou (2008). Nous présentons ici les résultats des datations radiocarbone des ours d'Ifri Oussaïd en les resituant dans leur contexte environnemental.

\section{2 - LE SITE D'IFRI OUSSAÏD}

\section{1 - SITUATION GÉOGRAPHIQUE ET CADRE GÉOLOGIQUE}

La zone d'étude se situe dans la partie centrale du Moyen Atlas plissé (fig. 1) à moins de $10 \mathrm{~km}$ au sud-ouest de la ville de Boulemane. Les affleurements géologiques sont formés de terrains qui vont stratigraphiquement depuis le Trias jusqu'au Crétacé. Les terrains triasiques sont composés d'argilites silteuses rougeâtres gypso-salifères à intercalation de basaltes doléritiques altérés. Ces terrains sont surmontés en concordance angulaire par les terrains jurassiques carbonatés composés de dolomies du Lias inférieur. Sur cette série triasico-liasique repose en discordance angulaire une formation gréseuse carbonatée d'âge Crétacé. L'analyse structurale montre que cette zone a subi une tectonique polyphasée décrite entre autres par Charroud (1990), Fedan et Charroud (1992) et Arboleya et al. (2004).

Les formations dolomitiques du Lias inférieur et gréseuses du Crétacé ont subi une importante morphogenèse karstique. Celle-ci s'est traduite, entre autre, par la genèse d'une grotte large et profonde, nommée Ifri Oussaïd, et d'un relief ruiniforme bien développé correspondant, dans cette zone, à la Cité des Pierres (Benabdelhadi et al., 2008).

\section{2 - LE SITE D'IFRI OUSSAIID ET SON CONTEXTE ENVIRONNEMENTAL}

La grotte d'Ifri Oussaïd est située dans la vallée de Chaabat el-Melha, relevant de la commune rurale d'Essaf

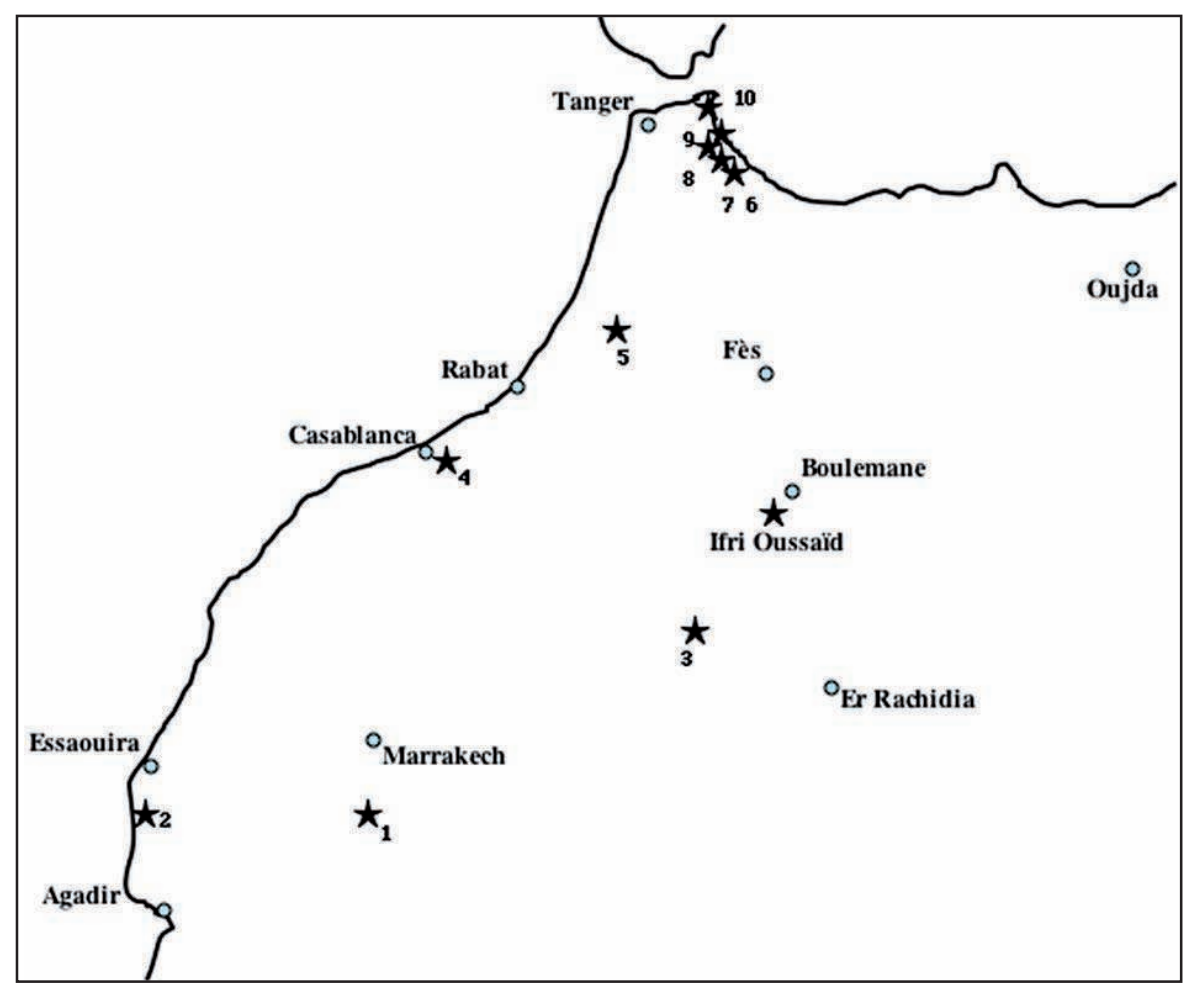

Fig. 1 : Carte des principaux sites holocènes à Ursidae du Maroc septentrional et position de la Grotte d'Ifri Oussaïd d'après Ouchaou (2008). Fig. 1: Location of the main Ursidae holocene sites in northern Morocco and location of Ifri Oussaïd cave from Ouchaou (2008). 
qui appartient à la province de Boulemane. La grotte qui se tient à une altitude de 2075 m, s'ouvre vers le sud-est sur un plateau ondulé, dénudé, et façonné dans les argilites et les basaltes triasiques. Du point de vue météorologique, nous ne disposons pas de données directes mais de celles de Tigalmamine ( $\left.32^{\circ} 54^{\prime} \mathrm{N}, 5^{\circ} 21^{\prime} \mathrm{W}, 1626 \mathrm{~m}\right)$ à moins de $40 \mathrm{~km}$ au sud ouest (Cheddadi et al., 1998). Les précipitations moyennes mensuelles de juin à septembre sont de $23 \mathrm{~mm}$, alors que celles des mois d'hiver (octobre à mai) sont de $126 \mathrm{~mm}$, soit $90 \%$ de la moyenne des précipitations annuelles totales $(930 \mathrm{~mm})$. La température moyenne de l'air pour juillet et janvier est de $20^{\circ} \mathrm{C}$ et $2,5^{\circ} \mathrm{C}$ respectivement (Martin, 1981).

Actuellement, autour d'Ifri Oussaïd, la végétation arborée est pratiquement inexistante à l'exception des berges d'oueds et de quelques thuyas sur les reliefs.

\section{3 - LES SONDAGES DE LA GROTTE}

$\mathrm{Au}$ cours des campagnes de fouilles et de prospection à la Cité des Pierres de 2005 à 2007, un sondage d'environ $30 \mathrm{~cm}$ de profondeur sur $2 \mathrm{~m}^{2}$ a été effectué dans la grotte (fig. 2). Sous deux planchers stalagmitiques séparés par 5 à $10 \mathrm{~cm}$ de sédiment marron ocre sont apparus des restes osseux dont la majorité (156) appartient à l'ours brun Ursus arctos (Ouchaou, 2008). La fouille de ces deux carrés et d'une petite partie du carré I11 a permis de récupérer plusieurs pièces déterminables (20 pièces en I11, 90 en I12 et 61 en I13). Entre les planchers P1 et P2 et sous le plancher P2, des charbons de bois ont pu être collectés pour datation radiocarbone. Les niveaux plus profonds n'ont pas été explorés, les fouilles ayant été suspendues pour protéger ce qui reste de la séquence des actes de vandalismes qui s'y sont déjà produits. Néanmoins, il n'est pas impossible de découvrir dans ces niveaux d'autres parties de squelettes d'ours en connexion. Plusieurs restes de microvertébrés: insectivores, rongeurs, chiroptères, amphibiens, etc. (Ouchaou, 2008) ont été récupérés au tamis. A la base de cette séquence, un radius d'ours a été utilisé pour la datation.

\section{4 - DATATIONS RADIOCARBONE}

Les charbons sont examinés à la loupe binoculaire afin d'éliminer d'éventuels contaminants (poils, fibres, radicelles...). Une quantité suffisante de l'échantillon est prélevée afin de procéder au traitement Acide, Base, Acide (A, B, A). Les charbons sont ensuite brûlés dans un courant d'oxygène ou chauffés quelques heures à $800{ }^{\circ} \mathrm{C}$ avec de l'oxyde de cuivre $(\mathrm{CuO})$. Le $\mathrm{CO}_{2}$ obtenu est ensuite réduit avec de l'hydrogène en présence de fer

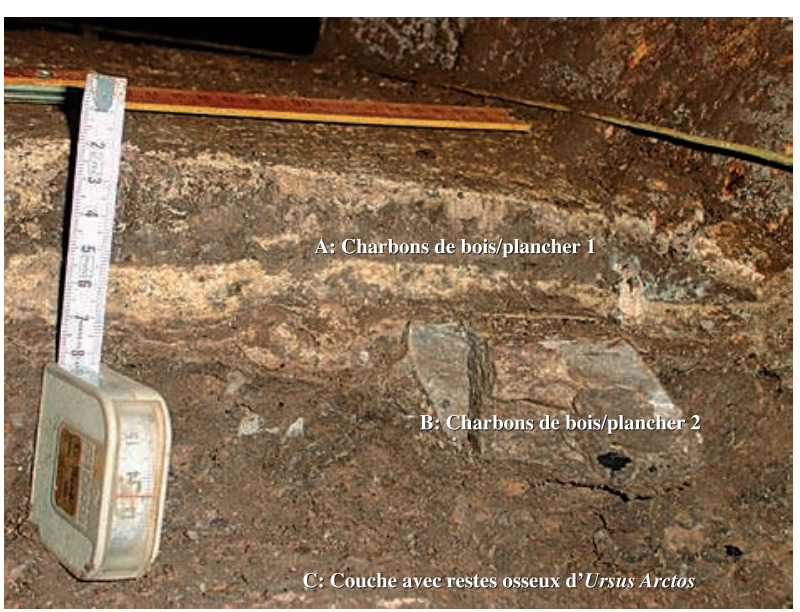

Fig. 2 : Coupe du sondage de la grotte d'Ifri Oussaïd avec les plancher stalagmitiques $\mathbf{P 1}$ et $\mathbf{P} 2$.

Fig. 2: Cross-section of the test pit from Ifri Oussaïd cave with stalagmitic floor Plet P2.

pour obtenir du graphite (Arnold et al., 1989; TisnératLaborde et al., 2001).

Les ossements réduits en poudre sont traités avec de l'acide chlorhydrique $(\mathrm{HCl} 0,5 \mathrm{M})$ pour éliminer carbonates et acides fulviques. Le résidu colloïdal restant est rincé à l'eau Milli-Q jusqu'à neutralité, puis $50 \mathrm{mg}$ de ninhydrine (2,2-dihydroxyñ1, 3-indandione) dans $2 \mathrm{ml}$ d'un tampon de citrate de sodium $(\mathrm{pH}=4,8)$ sont ajoutés au résidu qui est chauffé $10 \mathrm{mn}$ à $100^{\circ} \mathrm{C}$. La ninhydrine réagit spécifiquement avec les acides aminés pour obtenir le $\mathrm{CO}_{2}$ représentatif du collagène de l'ossement qui sera réduit comme indiqué précédemment (voir Nelson, 1991 et Tisnérat-Laborde et al. (2003) qui décrivent en détail le mode opératoire).

Des pastilles de graphite ou cibles sont préparées pour la mesure de l'activité ${ }^{14} \mathrm{C}$ qui s'effectue par spectrométrie de masse par accélération (SMA) à Saclay (91) au Laboratoire de Mesure du Carbone 14 (ARTEMIS).

Les âges radiocarbone sont calculés selon Mook \& Van der Plicht (1999) en corrigeant des fractionnements à partir de la mesure du rapport ${ }^{13} \mathrm{C} /{ }^{12} \mathrm{C}$ sur ARTEMIS. Ce $\delta^{13} \mathrm{C}$ inclut le fractionnement biologique et celui survenant aussi bien pendant la préparation des échantillons que pendant la mesure SMA. Il ne peut donc pas être considéré comme représentatif de la composition isotopique réelle du carbone $\left(\delta^{13} \mathrm{C}\right)$ de l'échantillon. Les intervalles de dates calibrées ont été calculés en utilisant le logiciel CALIB 5.1 (Stuiver \& Reimer, 1993) au niveau de confiance 95,4 \% (2 sigma). Les résultats sont reportés dans le tableau 1.

Les charbons sous les planchers stalagmitiques P1 et $\mathrm{P} 2$ donnent des âges voisins de respectivement $5220 \pm 30$

\begin{tabular}{|c|c|c|c|c|c|}
\hline N'Laboratoire & Localisation & Nature & $\begin{array}{c}\text { Age conventionnel B.P. } \\
\text { (ans 14C B.P. })\end{array}$ & $\begin{array}{c}\text { 13C } \\
(\% \circ)\end{array}$ & $\begin{array}{c}\text { Intervalle de dates Calibrées } \\
(\text { Cal BP })(2 \mathrm{sigma})\end{array}$ \\
\hline SacA-10205 & entre P1 et P2 & Charbons & $\mathbf{5 2 2 0 \pm 3 0}$ & $-28,8$ & $5913-6171$ \\
Gif-12309/SacA-10204 & sous P2 & Charbons & $5865 \pm 30$ & $-24,8$ & $6572-6775$ \\
GifA-80184/SacA12263 & sous P2 & Radius de Ursus a. & $\mathbf{7 3 0 0 \pm 4 0}$ & $-42,1$ & $8019-8180$ \\
\hline
\end{tabular}

Tab. 1 : Résultats des datations ${ }^{14} \mathrm{C}$ des charbons de bois et ossement de la Grotte d'Ifri Oussaïd.

Tab. 1: Results of ${ }^{14} \mathrm{C}$ datings of charcoals and bone collagen from d'Ifri Oussaïd cave. 
et $5865 \pm 30$ ans ${ }^{14} \mathrm{C} \mathrm{BP}$, soit $6171-5913$ et $6775-6572$ cal. BP autrement dit le VII ${ }^{\mathrm{e}}$ millénaire avant le présent. Le radius d'ours a fourni une date plus ancienne 7300 \pm 40 ans ${ }^{14} \mathrm{C}$ BP, soit $8180-8019$ cal. BP.

\section{3 - DISCUSSION}

Ces datations permettent de replacer la présence d'Ursus arctos dans un contexte climatique et environnemental depuis 8000 ans cal. BP. Le site d'Ifri Oussaïd et le lac de Tigalmamine sont distants de moins de $40 \mathrm{~km}$ à des altitudes comparables. Nous disposons pour Tigalmamine des travaux de Lamb \& van der Kaars (1995) et Lamb et al. (1989, 1991, 1995) concernant l'évolution de l'environnement végétal, et de ceux de Cheddadi et al. (1998) pour les paramètres climatiques tels que températures et précipitations pour la période holocène.

La présence d'Ursus arctos apparaît contemporaine de la zone 2 du diagramme pollinique de Tigalmamine qui correspond à une période de niveau relatif bas pour le lac. La végétation est dominée par le chêne (Quercus rotundifolia/Quercus ilex), le chêne canarien (Quercus canariensis) à feuilles caduques, et les graminées sont largement dominantes sur l'armoise et les chenopodiacées; le cèdre et le pin sont pratiquement absents. Une première ouverture du paysage au début de la zone 3 vers 5800 ans ${ }^{14} \mathrm{C}$ BP se traduit par une baisse du pourcentage de Quercus rotundifolia en parallèle de l'augmentation des graminées. Vers 3500 ans ${ }^{14} \mathrm{C}$ BP, le pourcentage de Quercus canariensis diminue sensiblement alors que le cèdre (Cedrus atlantica) semble être en expension.

En appliquant la méthode des analogues actuels à ces données polliniques, Cheddadi et al. (1998) estiment des températures hivernales de $6^{\circ} \mathrm{C}$ (janvier) et estivales de $24^{\circ} \mathrm{C}$ (juillet), soit environ $1{ }^{\circ} \mathrm{C}, 2,5^{\circ} \mathrm{C}$ de plus qu'actuellement; les précipitations moyennes estimées sont de $730 \mathrm{~mm}$ et sont inférieures de $70 \mathrm{~mm}$ par rapport à l'actuel. Ces estimations montrent que les conditions climatiques il y a 7300 ans ${ }^{14} \mathrm{C}$ BP diffèrent peu de l'actuel et que les variations au cours de l'Holocène présentent une gamme de variations étroites de $\pm 100 \mathrm{~mm}$ pour les précipitations et de $\pm 1,5$ et $\pm 1^{\circ} \mathrm{C}$ pour les températures de janvier et juillet respectivement. Le grand changement est l'absence autour du site d'Ifri Oussaïd de végétation arborée actuellement qui suggère des causes du changement plus anthropiques que climatiques.

Au-dessus du niveau à Ursus arctos, entre 5,2 et $5,8 \mathrm{ka}{ }^{14} \mathrm{C} \mathrm{BP}$, les planchers stalagmitiques indiquent une période plus humide en accord avec les observations de la zone 3 du diagramme pollinique qui en plus des taxons déjà énumérés pour la zone 2 indiquent l'apparition franche du pin et du cèdre. Les estimations de Cheddadi et al. (1998) évaluent les précipitations et températures voisines de ce qu'elles sont actuellement, les températures hivernales étant néanmoins supérieures de $0,5^{\circ} \mathrm{C}$.

Entre 6 et $2 \mathrm{ka}{ }^{14} \mathrm{C} \mathrm{BP}$, la situation restera stable aussi bien du point de vue climatique que du couvert végétal avec, néanmoins, une décroissance légère du chêne cana- rien au profit du cèdre. A partir de $2 \mathrm{ka}{ }^{14} \mathrm{C} \mathrm{BP}$, le chêne canarien disparaît rapidement sous la pression anthropique et l'ouverture des paysages aux activités pastorales (Cheddadi et al., 1998). Cette période, avec des températures (janvier et juillet) stables et proches de l'actuel, enregistre une légère baisse des précipitations également notée plus au nord dans les dépôts de la Dayet Afourgagh

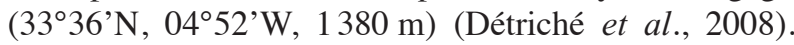
La stabilité des paramètres température et précipitation durant ces 6000 ans plaide peu pour une extinction de l'ours due à des causes climatiques. Malgré quelques indices d'un début d'anthropisation au nord du Maroc vers 4 ka BP (El Amrani et al., 2008) ce n'est seulement qu'à partir de $2 \mathrm{ka} \mathrm{BP}$ que ses effets sont notables au Maroc (Ballouche, 2003) et génèrent des conditions de vie très défavorables pour les ursidés.

\section{4 - CONCLUSION}

Ces résultats placent Ifri Oussaïd comme l'un des sites datés à ursidés holocènes les plus importants de l'Afrique du Nord. La présence de Ursus arctos dans le Moyen Atlas y est maintenant bien établie autour de 7300 ans ${ }^{14} \mathrm{C}$ BP (8100 cal. BP) dans des conditions climatiques proches de celles de l'actuel pour ce qui est des précipitations et des températures moyennes. Ces restes, d'ours de petite taille, avec ceux d'El Ksiba (Balloy \& Ennouchi, 1950) sont les plus importants connus dans l'Holocène des chaînes atlasiques marocaines. La stabilité de ces conditions et du couvert végétal jusqu'à $2 \mathrm{ka}{ }^{14} \mathrm{C} \mathrm{BP}$ suggère que la disparition d'Ursus arctos dans le Moyen Atlas n'est pas due directement à des causes climatiques. L'ouverture des paysages sous la pression anthropique à partir de $2 \mathrm{ka}{ }^{14} \mathrm{C} \mathrm{BP}$ apparaît plus déterminante pour l'extinction d'Ursus arctos.

\section{REMERCIEMENTS}

Ce travail a pu être engagé grâce au soutien d'une convention de collaboration CNRST-Maroc/CNRSFrance $\mathrm{N}^{\circ} 21058$ «Préhistoire et paléo-environnement quaternaires dans le Moyen Atlas, Maroc» et au PROTARS II marocain $\mathrm{n}^{\circ} \mathrm{P} 32 / 12$ «Recherches sur la préhistoire et la géologie du Quaternaire pour le développement socio-culturel de la région du Saiss et du Moyen Atlas». Nous remercions le Pr H. Aouraghe et un reviewer anonyme pour leurs remarques critiques et constructives.

\section{RÉFÉRENCES BIBIOGRAPHIQUES}

ARAMBOURG C., 1933 - Révision des ours fossiles de l'Afrique du Nord. Annales du Muséum d'Histoire Naturelle de Marseille, 25, 247-301.

ARBOLEYA M., TEIXELL A., CHARROUD M. \& JULIVERT M., 2004 - A structural transect through the High and Middle Atlas of Morocco. Journal of African Earth Sciences, 39 (3-5), 319-327.

ARNOLD M., BARD E., MAURICE P., VALLADAS H. \& DUPLESSY J.-C., $1989-{ }^{14} \mathrm{C}$ dating with the Gif-sur-Yvette Tande- 
tron accelerator: status report and study of isotopic fractionation in the sputter ion source. Radiocarbon, 31 (3), 284-291.

BALLOUCHE A., 2003 - La dimension anthropique de l'histoire de la végétation holocène au Maghreb. In Le peuplement ancien de l'Algérie: sa place dans le contexte Méditerranéen, Paris, 28-29 novembre 2003. 6 p. http://halshs.archives-ouvertes.fr/halshs00123883 .

BALLOY M., \& ENNOUCHI E., 1950 - Sur la découverte de six ours à El Ksiba. Bulletin de la Société des Sciences Naturelles du Maroc, 46, 103-108.

BENABDELHADI M., OUJAA A., CHARROUD M., OUCHAOU B., ZOUHRI S., BOUDAD L., BINOU K., SIMONIS C., PUCCINI D., \& FONTUGNE M., 2008 - Nouveaux sites préhistoriques dans le moyen Atlas marocain: résultats des premières recherches. In H. Aouraghe, H. Haddoumi \& K. El Hammouti (eds.), Le Quaternaire marocain dans son contexte méditerranéen. Actes de la quatrième rencontre des quaternaristes marocains (RQM4), Oujda, 15-17 novembre 2007. Volume 2: Paléontologie, Taphonomie et Préhistoire. Publications de la Faculté des Sciences d'Oujda, Oujda, 345-355.

BENABDELHADI M., CHARROUD M., OUJAA A., SBIHI ALAOUI F.Z., \& FALGUÈRES C., 2005 - Recherches préhistoriques dans le moyen Atlas. In Actes de la $3^{e}$ rencontre des quaternaristes marocains (RQM3), "Paléo-environnements quaternaires et cultures préhistoriques du Maroc », Marrakech, 15-17 novembre 2005. Publications de la Faculté des Sciences de Semlalia, Marrakech, $11 \mathrm{p}$.

CHARROUD M., 1990 - Evolution géodynamique de la partie SudOuest du Moyen Atlas durant le passage Jurassique-Crétacé, le Crétacé et le Paléogène: un exemple d'évolution intraplaque. Thèse de Doctorat, Université Mohammed V, Rabat, $234 \mathrm{p}$.

CHEDDADI R., LAMB H., GUIOT J. \& VAN DER KAARS S. 1998 - Reconstruction of the Holocene climatic events using a pollen record from Tigalmamine lake, Morocco: Relationships to global climate change. Climate Dynamics, 14 (12), 883-890.

DÉTRICHÉ S., BRÉHÉRET J.-G., ZARKI H., KARRAT L., MACAIRE J.-J., \& FONTUGNE M., 2008 - Late Holocene palaeohydrology of lake Afourgagh (Middle-Atlas, Morocco) from deposit geometry and facies. Bulletin de la Société géologique de France, 179 (1), 41-50.

EL AMRANI M., MACAIRE J.-J., ZARKI H., BRÉHÉRET J.-G., \& FONTUGNE M., 2008 - High Morpho-sedimentary reactivity of the lower Kert River (North Eastern Morocco) to palaeoenvironment change during the Holocene. Comptes Rendus Géoscience, 340 (8), 533-542.

ENNOUCHI E., 1957 - Les Ursidés marocains. Bulletin de la Société des Sciences Naturelles du Maroc, 37, 201-224.

FEDAN B. \& CHARROUD M., 1992 - Place des formations détritiques rouges jurassicocrétacées dans l'évolution géodynamique du Moyen Atlas. Notes et Mémoires du Service géologique. Maroc, 366, 461-462.
GERAADS D., 1997 - Carnivores du Pliocène terminal d'Ahl Al Oughlam (Casablanca, Maroc). Geobios, 30 (1), 127-164.

HAMDINE W., THEVENOT M. \& MICHAUX J., 1998 - Histoir récente de l'ours brun au Maghreb. Comptes Rendus de l'Académie des Sciences. Série 3, Sciences de la vie, 321 (7), 565-570.

LAMB H.F., EICHER U. \& SWITSUR V.R., 1989 - An 18,000-yea of vegetation, lake-level and climatic change from Tigalmamine, Middle Atlas, Morocco. Journal of Biogeography. 16 (1), 65-74.

LAMB H.F., DAMBLON F. \& MAXTED R.W., 1991 - Human impact on the vegetation of the midlle Atlas, Morocco, during the last 5000 years. Journal of Biogeography, $\mathbf{1 8}$ (5), 519- 532

LAMB H.F., GASSE F., BENKADDOUR A., EL HAMOUTI N., VAN DER KAARS S., PERKINS W.T., PEARCE N.J. \& ROBERTS C.N., 1995 - Relation between century scale Holocene arids intervals in tropical and temperates zones. Nature, 373 (6510), 134-137.

LAMB H.F. \& VAN DER KAARS S., 1995 - Vegetational response to Holocene climatic change: pollen and palaeolimnologic data from the Middle Atlas, Morocco. The Holocene, 5 (4), 400-408.

MARTIN J., 1981 - Le Moyen Atlas central étude géomorphologique. Notes et Mémoires du Service géologique, 258 bis, 1-447.

MICHAUX J., \& THEVENOT M., 2007 - Histoire récente de l'ours brun (Ursus arctos, Linné, 1758) au Maghreb. Contribution des fossiles d'Algérie. Mésogée, 63, 43-49.

MOOK W.G., \& VAN DER PLICHT J., 1999 - Reporting ${ }^{14} \mathrm{C}$ Activities and Concentrations. Radiocarbon, 41 (3), 227-239.

NELSON D.E., 1991 - A new method for carbon isotopic analysis of protein. Science, 251 (4993), 552-554.

OUCHAOU B., 2008 - Mise à jour de la liste des gisements à Ursidé dans l'Holocène marocain et données ostéométriques sur l'ours d'Ifri Oussaïd (Moyen Atlas). In H. Aouraghe, H. Haddoumi \& K El Hammouti (eds.), Le Ouaternaire marocain dans son contexte méditerranéen. Actes de la quatrième rencontre des quaternaristes marocains (RQM4), Oujda, 15-17 novembre 2007. Volume 2: Paléontologie, Taphonomie et Préhistoire. Publications de la Faculté des Sciences d'Oujda, Oujda, 265-279.

OUCHAOU B., \& AMANI F., 2002 - Les Carnivores des gisements néolithiques et protohistoriques du nord du Maroc. Quaternaire, 13 (1), 79-87.

STUIVER M., \& REIMER P.J., 1993 - Extended ${ }^{14} \mathrm{C}$ data base and revised Calib $3.0{ }^{14} \mathrm{C}$ age calibration programme. Radiocarbon, 35 (1), 215-230.

TISNÉRAT-LABORDE N., POUPEAU J.-J., TANNAU J.-F., \& PATERNE M., 2001 - Development of a semi-automated system for routine preparation of carbonate sample. Radiocarbon, 43 (2A), 299-304.

TISNÉRAT-LABORDE N., VALLADAS H., KALTNECKER E., \& ARNOLD M., 2003 - AMS radiocarbon dating of bones at LSCE. Radiocarbon, 45 (3), 409-419. 